\title{
Some identities of degenerate Euler polynomials associated with degenerate Bernstein polynomials
}

\author{
Won Joo Kim ${ }^{1 *}$, Dae San Kim², Han Young Kim ${ }^{3}$ and Taekyun Kim³
}

\section{"Correspondence:}

wjookim@khu.ac.kr

'Department of Applied

Mathematics, Kyunghee University,

Yongin-si, Republic of Korea

Full list of author information is

available at the end of the article

\begin{abstract}
In this paper, we investigate some properties and identities for degenerate Euler polynomials in connection with degenerate Bernstein polynomials by means of fermionic $p$-adic integrals on $\mathbb{Z}_{p}$ and generating functions. In addition, we study two variable degenerate Bernstein polynomials and the degenerate Bernstein operators.
\end{abstract}

MSC: 11B83; 11580

Keywords: Degenerate Euler polynomials; Degenerate Bernstein polynomials

\section{Introduction}

Let $p$ be a fixed odd prime number. Throughout this paper, $\mathbb{Z}_{p}, \mathbb{Q}_{p}$ and $\mathbb{C}_{p}$, will denote the ring of $p$-adic integers, the field of $p$-adic rational numbers and the completion of algebraic closure of $\mathbb{Q}_{p}$, respectively. Let $v_{p}$ be the normalized exponential valuation of $\mathbb{C}_{p}$ with $|p|_{p}=p^{-v_{p}(p)}=\frac{1}{p}$. For $\lambda \in \mathbb{C}_{p}$ with $|\lambda|_{p}<p^{-\frac{1}{p-1}}$, the degenerate Euler polynomials are defined by the generating function

$$
\frac{2}{(1+\lambda t)^{\frac{1}{\lambda}}+1}(1+\lambda t)^{\frac{x}{\lambda}}=\sum_{n=0}^{\infty} \mathcal{E}_{n, \lambda}(x) \frac{t^{n}}{n !} \quad(\text { see }[1,2]) .
$$

When $x=0, \mathcal{E}_{n, \lambda}=\mathcal{E}_{n, \lambda}(0)$ are called the degenerate Euler numbers. The degenerate exponential function is defined by

$$
e_{\lambda}^{x}(t)=(1+\lambda t)^{\frac{x}{\lambda}}=\sum_{n=0}^{\infty}(x)_{n, \lambda} \frac{t^{n}}{n !} \quad(\text { see }[6])
$$

where

$$
(x)_{0, \lambda}=1, \quad(x)_{n, \lambda}=x(x-\lambda)(x-2 \lambda) \cdots(x-(n-1) \lambda), \quad \text { for } n \geq 1 .
$$

From (1), we note that

$$
\mathcal{E}_{n, \lambda}(x)=\sum_{l=0}^{n}\left(\begin{array}{l}
n \\
l
\end{array}\right) \mathcal{E}_{l, \lambda}(x)_{n-l, \lambda} \quad(n \geq 0) .
$$

(c) The Author(s) 2019. This article is distributed under the terms of the Creative Commons Attribution 4.0 International License (http://creativecommons.org/licenses/by/4.0/), which permits unrestricted use, distribution, and reproduction in any medium, provided you give appropriate credit to the original author(s) and the source, provide a link to the Creative Commons license, and indicate if changes were made. 
Recently, Kim-Kim introduced the degenerate Bernstein polynomials given by

$$
\frac{(x)_{k, \lambda}}{k !} t^{k}(1+\lambda t)^{\frac{1-x}{\lambda}}=\sum_{n=k}^{\infty} B_{k, n}(x \mid \lambda) \frac{t^{n}}{n !} \quad(\text { see }[8-10]) .
$$

Thus, by (5), we get

$$
B_{k, n}(x \mid \lambda)= \begin{cases}\left(\begin{array}{l}
n \\
k
\end{array}\right)(x)_{k, \lambda}(1-x)_{n-k, \lambda}, & \text { if } n \geq k \\
0, & \text { if } n<k\end{cases}
$$

where $n, k$ are nonnegative integers.

Let $f$ be a continuous function on $\mathbb{Z}_{p}$. Then the degenerate Bernstein operator of order $n$ is given by

$$
\begin{aligned}
\mathbb{B}_{n, \lambda}(f \mid \lambda) & =\sum_{k=0}^{n} f\left(\frac{k}{n}\right)\left(\begin{array}{l}
n \\
k
\end{array}\right)(x)_{k, \lambda}(1-x)_{n-k, \lambda} \\
& =\sum_{k=0}^{n} f\left(\frac{k}{n}\right) B_{k, n}(x \mid \lambda) \quad(\text { see }[8,9,12-15,17-19]) .
\end{aligned}
$$

The fermionic $p$-adic integral on $\mathbb{Z}_{p}$ is defined by Kim as

$$
\int_{\mathbb{Z}_{p}} f(x) d \mu_{-1}(x)=\lim _{N \rightarrow \infty} \sum_{x=0}^{p^{N}-1} f(x)(-1)^{x} \quad(\text { see }[3,9]) .
$$

By (8), we get

$$
\int_{\mathbb{Z}_{p}} f(x+1) d \mu_{-1}(x)+\int_{\mathbb{Z}_{p}} f(x) d \mu_{-1}(x)=2 f(0) \quad(\text { see }[3,7,10,11,16]) .
$$

From (8), we note that

$$
\int_{\mathbb{Z}_{p}}(1+\lambda t)^{\frac{x+y}{\lambda}} d \mu_{-1}(y)=\frac{2}{(1+\lambda t)^{\frac{1}{\lambda}}+1}(1+\lambda t)^{\frac{x}{\lambda}}=\sum_{n=0}^{\infty} \mathcal{E}_{n, \lambda}(x) \frac{t^{n}}{n !} .
$$

On the other hand,

$$
\int_{\mathbb{Z}_{p}}(1+\lambda t)^{\frac{x+y}{\lambda}} d \mu_{-1}(y)=\sum_{n=0}^{\infty} \int_{\mathbb{Z}_{p}}(x+y)_{n, \lambda} d \mu_{-1}(y) \frac{t^{n}}{n !}
$$

By (10) and (11), we get

$$
\int_{\mathbb{Z}_{p}}(x+y)_{n, \lambda} d \mu_{-1}(y)=\mathcal{E}_{n, \lambda}(x) \quad(n \geq 0) \quad(\text { see }[8,9]) .
$$

The study of degenerate versions of some special polynomials and numbers began with the work of Carlitz on the degenerate Bernoulli and Euler polynomials and numbers in $[1,2]$. As a continuation of this initiative of Carlitz, Kim and his colleagues have been 
introducing various degenerate special polynomials and numbers and investigating their properties, some identities related to them and their applications. This research has been carried out by means of generating functions, combinatorial methods, umbral calculus, $p$ adic analysis and differential equations (see $[8,9]$ and the references therein). Here, along the same line and by virtue of fermionic $p$-adic integrals on $\mathbb{Z}_{p}$ and generating functions, we investigate some properties and identities for degenerate Euler polynomials related to degenerate Bernstein polynomials. In addition, we study two variable degenerate Bernstein polynomials and the degenerate Bernstein operators.

\section{Degenerate Euler and Bernstein polynomials}

From (1), we note that

$$
\begin{aligned}
2 & =\sum_{n=0}^{\infty}\left(\sum_{m=0}^{n}\left(\begin{array}{c}
n \\
m
\end{array}\right) \mathcal{E}_{m, \lambda}(1)_{n-m, \lambda}+\mathcal{E}_{n, \lambda}\right) \frac{t^{n}}{n !} \\
& =\sum_{n=0}^{\infty}\left(\mathcal{E}_{n, \lambda}(1)+\mathcal{E}_{n, \lambda}\right) \frac{t^{n}}{n !} .
\end{aligned}
$$

Comparing the coefficients on both sides of (13), we have

$$
\mathcal{E}_{n, \lambda}(1)+\mathcal{E}_{n, \lambda}=2 \delta_{0, n} \quad(n, k \geq 0)
$$

where $\delta_{n, k}$ is the Kronecker symbol.

By (1), we easily get

$$
\mathcal{E}_{n, \lambda}(1-x)=(-1)^{n} \mathcal{E}_{n,-\lambda}(x) \quad(n \geq 0) .
$$

From (1), (4) and (14), we note that

$$
\begin{aligned}
\mathcal{E}_{n, \lambda}(2) & =\sum_{l=0}^{n}\left(\begin{array}{l}
n \\
l
\end{array}\right) \mathcal{E}_{l, \lambda}(1)(1)_{n-l, \lambda} \\
& =(1)_{n, \lambda}+\sum_{l=1}^{n}\left(\begin{array}{l}
n \\
l
\end{array}\right) \mathcal{E}_{l, \lambda}(1)(1)_{n-l, \lambda} \\
& =2(1)_{n, \lambda}-\sum_{l=0}^{n}\left(\begin{array}{l}
n \\
l
\end{array}\right)(1)_{n-l, \lambda} \mathcal{E}_{l, \lambda} \\
& =2(1)_{n, \lambda}+\mathcal{E}_{n, \lambda},
\end{aligned}
$$

where $n$ is a positive integer.

Therefore, by (16), we obtain the following theorem.

Theorem 2.1 For $n \in \mathbb{N}$, we have

$$
\mathcal{E}_{n, \lambda}(2)=2(1)_{n, \lambda}+\mathcal{E}_{n, \lambda} .
$$


Note that

$$
(1-x)_{n, \lambda}=(-1)^{n}(x-1)_{n,-\lambda} \quad(n \geq 0) .
$$

Therefore, by (12), (15) and (17), we easily get

$$
\begin{aligned}
\int_{\mathbb{Z}_{p}}(1-x)_{n, \lambda} d \mu_{-1}(x) & =(-1)^{n} \int_{\mathbb{Z}_{p}}(x-1)_{n,-\lambda} d \mu_{-1}(x) \\
& =\int_{\mathbb{Z}_{p}}(x+2)_{n, \lambda} d \mu_{-1}(x) .
\end{aligned}
$$

Therefore, by (18) and Theorem 2.1, we obtain the following theorem.

Theorem 2.2 For $n \in \mathbb{N}$, we have

$$
\int_{\mathbb{Z}_{p}}(1-x)_{n, \lambda} d \mu_{-1}(x)=\int_{\mathbb{Z}_{p}}(x+2)_{n, \lambda} d \mu_{-1}(x)=2(1)_{n, \lambda}+\int_{\mathbb{Z}_{p}}(x)_{n, \lambda} d \mu_{-1}(x) .
$$

Corollary 2.3 For $n \in \mathbb{N}$, we have

$$
(-1)^{n} \mathcal{E}_{n,-\lambda}(-1)=2(1)_{n, \lambda}+\mathcal{E}_{n, \lambda}=\mathcal{E}_{n, \lambda}(2) .
$$

By (4), we get

$$
\begin{aligned}
\mathcal{E}_{n, \lambda}(1-x) & =\sum_{l=0}^{n}\left(\begin{array}{l}
n \\
l
\end{array}\right)(1-x)_{n-l, \lambda} \mathcal{E}_{l, \lambda} \\
& =\sum_{l=0}^{n}\left(\begin{array}{l}
n \\
l
\end{array}\right)(x)_{l, \lambda}(1-x)_{n-l, \lambda} \frac{\mathcal{E}_{l, \lambda}}{(x)_{l, \lambda}} \\
& =\sum_{l=0}^{n} B_{l, n}(x \mid \lambda) \mathcal{E}_{l, \lambda} \frac{1}{(x)_{l, \lambda}} .
\end{aligned}
$$

Let

$$
\frac{1}{(x)_{l, \lambda}}=\frac{1}{x(x-\lambda)(x-2 \lambda) \cdots(x-(l-1) \lambda)}=\sum_{k=0}^{l-1} \frac{A_{k}}{x-k \lambda} \quad(l \in \mathbb{N}) .
$$

Then we have

$$
A_{k}=\lambda^{1-l} \prod_{\substack{i=0, i \neq k}}^{l-1}\left(\frac{1}{k-i}\right)=\lambda^{1-l} \frac{(-1)^{k-l-1}}{k !(l-1-k) !}=\frac{\lambda^{1-l}}{(l-1) !}\left(\begin{array}{c}
l-1 \\
k
\end{array}\right)(-1)^{k-l-1} .
$$

By (20) and (21), we get

$$
A_{k}=\frac{(-\lambda)^{1-l}}{(l-1) !}\left(\begin{array}{c}
l-1 \\
k
\end{array}\right)(-1)^{k}
$$


From (20) and (22), we have

$$
\frac{1}{(x)_{l, \lambda}}=\sum_{k=0}^{l-1} \frac{(-1)^{k}}{(l-1) !}\left(\begin{array}{c}
l-1 \\
k
\end{array}\right) \frac{(-\lambda)^{1-l}}{x-k \lambda} \quad(l \in \mathbb{N})
$$

By (19) and (20), we get

$$
\begin{aligned}
\mathcal{E}_{n, \lambda}(1-x) & =\sum_{l=0}^{n} B_{l, n}(x \mid \lambda) \mathcal{E}_{l, \lambda} \frac{1}{(x)_{l, \lambda}} \\
& =(1-x)_{n, \lambda}+\sum_{l=1}^{n} B_{l, n}(x \mid \lambda) \mathcal{E}_{l, \lambda} \frac{1}{(x)_{l, \lambda}} \\
& =(1-x)_{n, \lambda}+\sum_{l=1}^{n} B_{l, n}(x \mid \lambda) \mathcal{E}_{l, \lambda} \frac{(-\lambda)^{1-l}}{(l-1) !} \sum_{k=0}^{l-1}(-1)^{k}\left(\begin{array}{c}
l-1 \\
k
\end{array}\right) \frac{1}{x-k \lambda} .
\end{aligned}
$$

Therefore, by (24), we obtain the following theorem.

Theorem 2.4 For $n \geq 0$, we have

$$
\mathcal{E}_{n, \lambda}(1-x)=(1-x)_{n, \lambda}+\sum_{l=1}^{n} B_{l, n}(x \mid \lambda) \mathcal{E}_{l, \lambda} \frac{(-\lambda)^{1-l}}{(l-1) !} \sum_{k=0}^{l-1}(-1)^{k}\left(\begin{array}{c}
l-1 \\
k
\end{array}\right) \frac{1}{x-k \lambda} .
$$

Corollary 2.5 For $n \geq 0$, we have

$$
\mathcal{E}_{n, \lambda}(2)=(2)_{n, \lambda}-\sum_{l=1}^{n} B_{l, n}(-1 \mid \lambda) \mathcal{E}_{l, \lambda} \frac{(-\lambda)^{1-l}}{(l-1) !} \sum_{k=0}^{l-1}(-1)^{k}\left(\begin{array}{c}
l-1 \\
k
\end{array}\right) \frac{1}{1+k \lambda}
$$

For $k \in \mathbb{N}$, the higher order degenerate Euler polynomials are given by the generating function

$$
\left(\frac{2}{(1+\lambda t)^{\frac{1}{\lambda}}+1}\right)^{k}(1+\lambda t)^{\frac{x}{\lambda}}=\sum_{n=0}^{\infty} \mathcal{E}_{n, \lambda}^{(k)}(x) \frac{t^{n}}{n !} \quad(\text { see }[4,5])
$$

From (5) and (25), we note that

$$
\begin{aligned}
\sum_{n=0}^{\infty} \frac{1}{\left(\begin{array}{c}
n+k \\
n
\end{array}\right)} B_{k, n+k}(x \mid \lambda) \frac{t^{n}}{n !} & =(x)_{k, \lambda}(1+\lambda t)^{\frac{1-x}{\lambda}} \\
& =\frac{(x)_{k, \lambda}}{2^{k}} \sum_{l=0}^{k}\left(\begin{array}{l}
k \\
l
\end{array}\right)\left(\frac{2}{(1+\lambda t)^{\frac{1}{\lambda}}+1}\right)^{k}(1+\lambda t)^{\frac{1-x+l}{\lambda}} \\
& =\frac{(x)_{k, \lambda}}{2^{k}} \sum_{n=0}^{\infty}\left(\sum_{l=0}^{k}\left(\begin{array}{l}
k \\
l
\end{array}\right) \mathcal{E}_{n, \lambda}^{(k)}(1-x+l)\right) \frac{t^{n}}{n !}
\end{aligned}
$$

Therefore, by comparing the coefficients on both sides of (26), we obtain the following theorem. 
Theorem 2.6 For $n, k \in \mathbb{N}$, we have

$$
\frac{2^{k}}{\left(\begin{array}{c}
n+k \\
n
\end{array}\right)} B_{k, n+k}(x \mid \lambda)=(x)_{k, \lambda} \sum_{l=0}^{k}\left(\begin{array}{l}
k \\
l
\end{array}\right) \mathcal{E}_{n, \lambda}^{(k)}(1-x+l) .
$$

Let $f$ be a continuous function on $\mathbb{Z}_{p}$. For $x_{1}, x_{2} \in \mathbb{Z}_{p}$, we consider the degenerate Bernstein operator of order $n$ given by

$$
\begin{aligned}
\mathbb{B}_{n, \lambda}\left(f \mid x_{1}, x_{2}\right) & =\sum_{k=0}^{n} f\left(\frac{k}{n}\right)\left(\begin{array}{l}
n \\
k
\end{array}\right)\left(x_{1}\right)_{k, \lambda}\left(1-x_{2}\right)_{n-k, \lambda} \\
& =\sum_{k=0}^{n} f\left(\frac{k}{n}\right) B_{k, n}\left(x_{1}, x_{2} \mid \lambda\right),
\end{aligned}
$$

where

$$
B_{k, n}\left(x_{1}, x_{2} \mid \lambda\right)=\left(\begin{array}{l}
n \\
k
\end{array}\right)\left(x_{1}\right)_{k, \lambda}\left(1-x_{2}\right)_{n-k, \lambda},
$$

where $n, k$ are nonnegative integers.

Here, $B_{k, n}\left(x_{1}, x_{2} \mid \lambda\right)$ are called two variable degenerate Bernstein polynomials of degree $n$. From (28), we note that

$$
\begin{aligned}
\sum_{n=k}^{\infty} B_{k, n}\left(x_{1}, x_{2} \mid \lambda\right) \frac{t^{n}}{n !} & =\sum_{n=k}^{\infty}\left(\begin{array}{l}
n \\
k
\end{array}\right)\left(x_{1}\right)_{k, \lambda}\left(1-x_{2}\right)_{n-k, \lambda} \frac{t^{n}}{n !} \\
& =\sum_{n=k}^{\infty} \frac{\left(x_{1}\right)_{k, \lambda}\left(1-x_{2}\right)_{n-k, \lambda}}{k !(n-k) !} t^{n} \\
& =\frac{\left(x_{1}\right)_{k, \lambda}}{k !} t^{k} \sum_{n=0}^{\infty} \frac{\left(1-x_{2}\right)_{n, \lambda}}{n !} t^{n} \\
& =\frac{\left(x_{1}\right)_{k, \lambda}}{k !} t^{k}(1+\lambda t)^{\frac{1-x_{2}}{\lambda}} \\
& =\frac{\left(x_{1}\right)_{k, \lambda}}{k !} t^{k} e_{\lambda}^{1-x_{2}}(t) .
\end{aligned}
$$

Thus, by (29), we get

$$
\frac{\left(x_{1}\right)_{k, \lambda}}{k !} t^{k}(1+\lambda t)^{\frac{1-x_{2}}{\lambda}}=\sum_{n=k}^{\infty} B_{k, n}\left(x_{1}, x_{2} \mid \lambda\right) \frac{t^{n}}{n !}
$$

where $k$ is a nonnegative integer. By (28), we easily get

$$
\begin{aligned}
B_{k, n}\left(x_{1}, x_{2} \mid \lambda\right) & =\left(\begin{array}{l}
n \\
k
\end{array}\right)\left(1-\left(1-x_{1}\right)\right)_{n-(n-k), \lambda}\left(1-x_{2}\right)_{n-k, \lambda} \\
& =B_{n-k, n}\left(1-x_{2}, 1-x_{1} \mid \lambda\right) .
\end{aligned}
$$


Now, we observe that

$$
\begin{aligned}
\left(1-x_{2}-(n-k-1) \lambda\right) B_{k, n-1}\left(x_{1}, x_{2} \mid \lambda\right)+\left(x_{1}-(k-1) \lambda\right) B_{k-1, n-1}\left(x_{1}, x_{2} \mid \lambda\right) \\
=\left(1-x_{2}-(n-k-1) \lambda\right)\left(\begin{array}{c}
n-1 \\
k
\end{array}\right)\left(x_{1}\right)_{k, \lambda}\left(1-x_{2}\right)_{n-1-k, \lambda} \\
\quad+\left(x_{1}-(k-1) \lambda\right)\left(\begin{array}{c}
n-1 \\
k-1
\end{array}\right)\left(x_{1}\right)_{k-1, \lambda}\left(1-x_{2}\right)_{n-k, \lambda} \\
=\left(\begin{array}{l}
n \\
k
\end{array}\right)\left(x_{1}\right)_{k, \lambda}\left(1-x_{2}\right)_{n-k, \lambda}=B_{k, n}\left(x_{1}, x_{2} \mid \lambda\right) \quad(n, k \in \mathbb{N}) .
\end{aligned}
$$

Therefore, by (32), we obtain the following theorem.

Theorem 2.7 For $n, k \in \mathbb{N}$, we have

$$
\begin{aligned}
(1 & \left.-x_{2}-(n-k-1) \lambda\right) B_{k, n-1}\left(x_{1}, x_{2} \mid \lambda\right)+\left(x_{1}-(k-1) \lambda\right) B_{k-1, n-1}\left(x_{1}, x_{2} \mid \lambda\right) \\
& =B_{k, n}\left(x_{1}, x_{2} \mid \lambda\right) .
\end{aligned}
$$

If $f=1$, then we have, from (27),

$$
\begin{aligned}
\mathbb{B}_{n, \lambda}\left(1 \mid x_{1}, x_{2}\right) & =\sum_{k=0}^{n} B_{k, n}\left(x_{1}, x_{2} \mid \lambda\right)=\sum_{k=0}^{n}\left(\begin{array}{l}
n \\
k
\end{array}\right)\left(x_{1}\right)_{k, \lambda}\left(1-x_{2}\right)_{n-k, \lambda} \\
& =\left(1+x_{1}-x_{2}\right)_{n, \lambda} .
\end{aligned}
$$

If $f(t)=t$, then we also get from (27) that, for $n \in \mathbb{N}$ and $x_{1}, x_{2} \in \mathbb{Z}_{p}$,

$$
\begin{aligned}
\mathbb{B}_{n, \lambda}\left(t \mid x_{1}, x_{2}\right) & =\sum_{k=0}^{n} \frac{k}{n}\left(\begin{array}{l}
n \\
k
\end{array}\right)\left(x_{1}\right)_{k, \lambda}\left(1-x_{2}\right)_{n-k, \lambda} \\
& =\left(x_{1}\right)_{1, \lambda}\left(x_{1}+1-\lambda-x_{2}\right)_{n-1, \lambda} .
\end{aligned}
$$

Hence,

$$
\left(x_{1}\right)_{1, \lambda}=\frac{1}{\left(x_{1}+1-\lambda-x_{2}\right)_{n-1, \lambda}} \mathbb{B}_{n, \lambda}\left(t \mid x_{1}, x_{2}\right) .
$$

By the same method, we get

$$
\begin{aligned}
& \mathbb{B}_{n, \lambda}\left(t^{2} \mid x_{1}, x_{2}\right) \\
& \quad=\frac{1}{n}\left(x_{1}\right)_{1, \lambda}\left(1+x_{1}-\lambda-x_{2}\right)_{n-1, \lambda}+\frac{n-1}{n}\left(x_{1}\right)_{2, \lambda}\left(1+x_{1}-2 \lambda-x_{2}\right)_{n-2, \lambda} .
\end{aligned}
$$

Note that

$$
\lim _{n \rightarrow \infty}\left(\lim _{\lambda \rightarrow 0} \mathbb{B}_{n, \lambda}\left(t^{2} \mid x, x\right)\right)=\lim _{n \rightarrow \infty}\left(\frac{x}{n}+\frac{n-1}{n} x^{2}\right)=x^{2} .
$$


Now, we observe that

$$
\begin{aligned}
\sum_{k=1}^{n} \frac{\left(\begin{array}{l}
k \\
1
\end{array}\right)}{\left(\begin{array}{l}
n \\
1
\end{array}\right)} B_{k, n}\left(x_{1}, x_{2} \mid \lambda\right) & =\sum_{k=1}^{n}\left(\begin{array}{c}
n-1 \\
k-1
\end{array}\right)\left(x_{1}\right)_{k, \lambda}\left(1-x_{2}\right)_{n-k, \lambda} \\
& =\sum_{k=0}^{n-1}\left(\begin{array}{c}
n-1 \\
k
\end{array}\right)\left(x_{1}\right)_{k+1, \lambda}\left(1-x_{2}\right)_{n-1-k, \lambda} \\
& =\left(x_{1}\right)_{1, \lambda}\left(x_{1}+1-\lambda-x_{2}\right)_{n-1, \lambda} .
\end{aligned}
$$

Thus, by (37), we get

$$
\left(x_{1}\right)_{1, \lambda}=\frac{1}{\left(1+x_{1}-x_{2}-\lambda\right)} \sum_{n-1, \lambda} \sum_{k=1}^{n} \frac{\left(\begin{array}{l}
k \\
1
\end{array}\right)}{\left(\begin{array}{l}
n \\
1
\end{array}\right)} B_{k, n}\left(x_{1}, x_{2} \mid \lambda\right) .
$$

By the same method, we get

$$
\left(x_{1}\right)_{2, \lambda}=\frac{1}{\left(1+x_{1}-x_{2}-2 \lambda\right)} \sum_{n-2, \lambda} \sum_{k=2}^{n} \frac{\left(\begin{array}{l}
k \\
2
\end{array}\right)}{\left(\begin{array}{l}
n \\
2
\end{array}\right)} B_{k, n}\left(x_{1}, x_{2} \mid \lambda\right) .
$$

Continuing this process, we have

$$
\left(x_{1}\right)_{i, \lambda}=\frac{1}{\left(1+x_{1}-x_{2}-i \lambda\right)} \sum_{n-i, \lambda} \sum_{k=i}^{n} \frac{\left(\begin{array}{l}
k \\
i
\end{array}\right)}{\left(\begin{array}{l}
n \\
i
\end{array}\right)} B_{k, n}\left(x_{1}, x_{2} \mid \lambda\right) \quad(i \in \mathbb{N}) .
$$

Theorem 2.8 For $i \in \mathbb{N}$, we have

$$
(x)_{i, \lambda}=\frac{1}{\left(1+x_{1}-x_{2}-i \lambda\right)_{n-i, \lambda}} \sum_{k=i}^{n} \frac{\left(\begin{array}{l}
k \\
i
\end{array}\right)}{\left(\begin{array}{l}
n \\
i
\end{array}\right)} B_{k, n}\left(x_{1}, x_{2} \mid \lambda\right) \quad(i \in \mathbb{N}) .
$$

Taking the double fermionic $p$-adic integral on $\mathbb{Z}_{p}$, we get the following equation:

$$
\begin{aligned}
& \int_{\mathbb{Z}_{p}} \int_{\mathbb{Z}_{p}} B_{k, n}\left(x_{1}, x_{2} \mid \lambda\right) d \mu_{-1}\left(x_{1}\right) d \mu_{-1}\left(x_{2}\right) \\
& \quad=\left(\begin{array}{l}
n \\
k
\end{array}\right) \int_{\mathbb{Z}_{p}}\left(x_{1}\right)_{k, \lambda} d \mu_{-1}\left(x_{1}\right) \int_{\mathbb{Z}_{p}}\left(1-x_{2}\right)_{n-k, \lambda} d \mu_{-1}\left(x_{2}\right) .
\end{aligned}
$$

Therefore, by (39) and Theorem 2.2, we obtain the following theorem.

Theorem 2.9 For $n, k \geq 0$, we have

$$
\begin{aligned}
& \int_{\mathbb{Z}_{p}} \int_{\mathbb{Z}_{p}} B_{k, n}\left(x_{1}, x_{2} \mid \lambda\right) d \mu_{-1}\left(x_{1}\right) d \mu_{-1}\left(x_{2}\right) \\
& \quad= \begin{cases}\left(\begin{array}{l}
n \\
k
\end{array}\right) \mathcal{E}_{k, \lambda}\left(2(1)_{n-k, \lambda}+\mathcal{E}_{n-k, \lambda}\right), & \text { if } n>k, \\
\mathcal{E}_{n, \lambda}, & \text { if } n=k .\end{cases}
\end{aligned}
$$


We see from the symmetric properties of two variable degenerate Bernstein polynomials that, for $n, k \in \mathbb{N}$ with $n>k$,

$$
\begin{aligned}
\int_{\mathbb{Z}_{p}} \int_{\mathbb{Z}_{p}} B_{k, n}\left(x_{1}, x_{2} \mid \lambda\right) d \mu_{-1}\left(x_{1}\right) d \mu_{-1}\left(x_{2}\right) \\
=\sum_{l=0}^{k}\left(\begin{array}{l}
n \\
k
\end{array}\right)\left(\begin{array}{l}
k \\
l
\end{array}\right)(-1)^{k+l}(1)_{l, \lambda} \\
\quad \times \int_{\mathbb{Z}_{p}} \int_{\mathbb{Z}_{p}}\left(1-x_{1}\right)_{k-l,-\lambda}\left(1-x_{2}\right)_{n-k, \lambda} d \mu_{-1}\left(x_{1}\right) d \mu_{-1}\left(x_{2}\right) \\
=\left(\begin{array}{l}
n \\
k
\end{array}\right) \int_{\mathbb{Z}_{p}}\left(1-x_{2}\right)_{n-k, \lambda} d \mu_{-1}\left(x_{2}\right)\left\{(1)_{k, \lambda}+\sum_{l=0}^{k-1}\left(\begin{array}{l}
k \\
l
\end{array}\right)(-1)^{k+l}(1)_{l, \lambda} \mathcal{E}_{k-l,-\lambda}(2)\right\} \\
=\left(\begin{array}{l}
n \\
k
\end{array}\right) \mathcal{E}_{n-k, \lambda}(2)\left\{(1)_{k, \lambda}+\sum_{l=0}^{k-1}\left(\begin{array}{l}
k \\
l
\end{array}\right)(-1)^{k+l}(1)_{l, \lambda} \mathcal{E}_{k-l,-\lambda}(2)\right\} .
\end{aligned}
$$

Therefore, by Theorem 2.9 and (40), we obtain the following theorem.

Theorem 2.10 For $k \in \mathbb{N}$, we have

$$
\mathcal{E}_{k, \lambda}=(1)_{k, \lambda}+\sum_{l=0}^{k-1}\left(\begin{array}{l}
k \\
l
\end{array}\right)(-1)^{k+l}(1)_{l, \lambda}\left(\mathcal{E}_{k-l,-\lambda}+2(1)_{k-l,-\lambda}\right) .
$$

Note that

$$
\begin{aligned}
& \sum_{l=0}^{k-1}\left(\begin{array}{l}
k \\
l
\end{array}\right)(-1)^{k+l}(1)_{l, \lambda}(1)_{k-l,-\lambda} \\
& =(-1)^{k}\left(\sum_{l=0}^{k}\left(\begin{array}{l}
k \\
l
\end{array}\right)(-1)_{l,-\lambda}(1)_{k-l,-\lambda}-(-1)_{k,-\lambda}\right) \\
& =(-1)^{k}\left((0)_{k,-\lambda}-(-1)_{k,-\lambda}\right) \\
& =-(1)_{k, \lambda} .
\end{aligned}
$$

Corollary 2.11 For $k \in \mathbb{N}$, we have

$$
\mathcal{E}_{k, \lambda}=-(1)_{k, \lambda}+\sum_{l=0}^{k-1}\left(\begin{array}{l}
k \\
l
\end{array}\right)(-1)^{k+l}(1)_{l, \lambda} \mathcal{E}_{k-l,-\lambda} .
$$

\section{Conclusions}

In $[1,2]$, Carlitz initiated the study of degenerate versions of some special polynomials and numbers, namely the degenerate Bernoulli and Euler polynomials and numbers. Here we would like to draw the attention of the reader to the fact that Kim et al. have introduced various degenerate polynomials and numbers and investigating their properties, some identities related to them and their applications by means of generating functions, combinatorial methods, umbral calculus, $p$-adic analysis and differential equations (see $[8,9]$ and the references therein). It is amusing that this line of study led them even to 
the introduction of degenerate gamma functions and degenerate Laplace transforms (see [7]). These already demonstrate that studying various degenerate versions of known special numbers and polynomials can be very promising and rewarding. Furthermore, we can hope that many applications will be found not only in mathematics but also in sciences and engineering.

In this paper, we investigated some properties and identities for degenerate Euler polynomials in connection with degenerate Bernstein polynomials and operators which were recently introduced as degenerate versions of the classical Bernstein polynomials and operators. This has been done by means of fermionic $p$-adic integrals on $\mathbb{Z}_{p}$ and generating functions. In addition, we studied two variable degenerate Bernstein polynomials and the degenerate Bernstein operators.

\section{Acknowledgements}

The fourth author's work in this paper was conducted during the sabbatical year of Kwangwoon University in 2018.

\section{Funding}

This research received no external funding.

\section{Competing interests}

The authors declare that they have no competing interests.

\section{Authors' contributions}

All authors contributed equally to the manuscript and typed, read, and approved the final manuscript.

\section{Author details}

${ }^{1}$ Department of Applied Mathematics, Kyunghee University, Yongin-si, Republic of Korea. ${ }^{2}$ Department of Mathematics, Sogang University, Seoul, Republic of Korea. ${ }^{3}$ Department of Mathematics, Kwnagwoon University, Seoul, Republic of Korea.

\section{Publisher's Note}

Springer Nature remains neutral with regard to jurisdictional claims in published maps and institutional affiliations.

Received: 28 March 2019 Accepted: 27 May 2019 Published online: 04 June 2019

\section{References}

1. Carlitz, L.: A degenerate Staudt-Clausen theorem. Arch. Math. 7, 28-33 (1956)

2. Carlitz, L.: Degenerate Stirling, Bernoulli and Eulerian numbers. Util. Math. 15, 51-88 (1979)

3. Choi, J.: A note on $p$-adic integrals associated with Bernstein and $q$-Bernstein polynomials. Adv. Stud. Contemp. Math. (Kyungshang) 21(2), 133-138 (2011)

4. Kim, D.S., Kim, T.: Some identities of degenerate Euler polynomials arising from $p$-adic fermionic integrals on $\mathbb{Z}_{p}$. Integral Transforms Spec. Funct. 26, 295-302 (2015)

5. Kim, D.S., Kim, T.: Higher-order degenerate Euler polynomials. Appl. Math. Sci. 9(2), 57-73 (2015)

6. Kim, T., Kim, D.S.: Symmetry of power sum polynomials and multivariate fermionic $p$-adic invariant integral on $\mathbb{Z}_{p}$. Russ. J. Math. Phys. 16(1), 93-96 (2009)

7. Kim, T., Kim, D.S.: Degenerate Laplace transform and degenerate gamma functions. Russ. J. Math. Phys. 24(2), 241-248 (2017)

8. Kim, T., Kim, D.S.: Degenerate Bernstein polynomials. Rev. R. Acad. Cienc. Exactas Fís. Nat., Ser. A Mat. (2018). https://doi.org/10.1007/s13398-018-0594-9

9. Kim, T. Kim, D.S.: Some identities on degenerate Bernstein polynomials and degenerate Euler polynomials. Mathematics 7(1), 47 (2019) https://doi.org/10.3390/math7010047

10. Kim, T., Kim, D.S., Jang, G.-W., Kwon, J.: A note on degenerate Bernstein polynomials. J. Inequal. Appl. 2019(2019), 129 (2019)

11. Kurt, V: Some relation between the Bernstein polynomials and second kind Bernoulli polynomials. Adv. Stud. Contemp. Math. (Kyungshang) 23(1), 43-48 (2013)

12. Ostrovska, S.: On the $q$-Bernstein polynomials. Adv. Stud. Contemp. Math. (Kyungshang) 11(2), 193-204 (2005)

13. Siddiqui, M.A., Agrawal, R.R., Gupta, N.: On a class of modified new Bernstein operators. Adv. Stud. Contemp. Math. (Kyungshang) 24(1), 97-107 (2014)

14. Simsek, B., Yardimci, A.: Using Bezier curves in medical applications. Filomat 30(4), 937-943 (2016)

15. Simsek, Y.: A new class of polynomials associated with Bernstein and beta polynomials. Math. Methods Appl. Sci. 37(5), 676-685 (2014)

16. Simsek, Y: Analysis of the Bernstein basis functions; an approach to combinatorial sums involving binomial coefficients and Catalan numbers. Math. Methods Appl. Sci. 38(14), 3007-3021 (2015)

17. Simsek, Y: Combinatorial identities associated with Bernstein type basis functions. Filomat 30(7), 1683-1689 (2016) 
18. Simsek, Y.: Identities on the Changhee numbers and Apostol-type Daehee polynomials. Adv. Stud. Contemp. Math. (Kyungshang) 27(2), 199-212 (2017)

19. Simsek, Y.: Combinatorial inequalities and sums involving Bernstein polynomials and basis functions. J. Inequal. Spec. Funct. 8(3), 15-24 (2017)

Submit your manuscript to a SpringerOpen ${ }^{\odot}$ journal and benefit from:

- Convenient online submission

$\checkmark$ Rigorous peer review

- Open access: articles freely available online

- High visibility within the field

- Retaining the copyright to your article

Submit your next manuscript at $\gg$ springeropen.com 